\title{
Conservation laws and exact solutions for the generalized Ostrovsky equation using symmetry analysis
}

\author{
Sol Sáez ${ }^{1}$ \\ ${ }^{1}$ University of Cadiz
}

July 20, 2021

\begin{abstract}
In this work we consider a generalized Ostrovsky equation depending on two arbitrary functions and we make an in-depth study of this equation. We obtain the Lie symmetries which are admitted by this equation and some exact solutions as a periodic or solitary waves, obtained through ordinary and partial differential equations. Also, by means of the concept of multiplier, we obtain a wide range of conservation laws which preserve properties of the generalized Ostrovsky equation.
\end{abstract}

\section{Hosted file}

Conservation laws and exact solutions for the generalized Ostrovsky.pdf available at https : //authorea.com/users/426700/articles/531125-conservation-laws-and-exact-solutionsfor-the-generalized-ostrovsky-equation-using-symmetry-analysis 